\title{
Conley index in Hilbert spaces and a problem of Angenent and van der Vorst
}

\author{
by \\ Marek Izydorek (Gdańsk) and \\ Krzysztof P. Rybakowski (Rostock)
}

\begin{abstract}
In a recent paper [9] we presented a Galerkin-type Conley index theory for certain classes of infinite-dimensional ODEs without the uniqueness property of the Cauchy problem. In this paper we show how to apply this theory to strongly indefinite elliptic systems. More specifically, we study the elliptic system

$$
\begin{array}{rlrl}
-\Delta u & =\partial_{v} H(u, v, x) & & \text { in } \Omega, \\
-\Delta v & =\partial_{u} H(u, v, x) & & \text { in } \Omega, \\
u=0, \quad v=0 & & \text { in } \partial \Omega,
\end{array}
$$

on a smooth bounded domain $\Omega$ in $\mathbb{R}^{N}$ for "-"-type Hamiltonians $H$ of class $C^{2}$ satisfying subcritical growth assumptions on their first order derivatives. As shown by Angenent and van der Vorst in [1], the solutions of $(A 1)$ are equilibria of an abstract ordinary differential equation
\end{abstract}

$$
\dot{z}=f(z)
$$

defined on a certain Hilbert space $E$ of functions $z=(u, v)$. The map $f: E \rightarrow E$ is continuous, but, in general, not differentiable nor even locally Lipschitzian.

The main result of this paper is a Linearization Principle which states that whenever $z_{0}$ is a hyperbolic equilibrium of $(A 2)$ then the Conley index of $\left\{z_{0}\right\}$ can be computed by formally linearizing $(A 2)$ at $z_{0}$.

As a particular application of the Linearization Principle we obtain an elementary, Conley index based proof of the existence of nontrivial solutions of $(A 1)$, a result previously established in [1] via Morse-Floer homology.

Further applications of our method to existence and multiplicity results for strongly indefinite systems appear in [3] and [10]. $58 \mathrm{E} 05$.

2000 Mathematics Subject Classification: Primary 37B30, 47J35; Secondary 34G20,

Key words and phrases: Galerkin-type Conley index, strongly indefinite elliptic systems, Linearization Principle, Morse-Floer homology.

Research of M. Izydorek supported by the Alexander von Humboldt Foundation. 
1. Introduction. Let $\Omega$ be a bounded domain in $\mathbb{R}^{N}$ with smooth boundary. Consider the following elliptic system:

$$
\begin{array}{rlrl}
-\Delta u & =\partial_{v} H(u, v, x) & & \text { in } \Omega, \\
-\Delta v & =\partial_{u} H(u, v, x) & & \text { in } \Omega, \\
u=0, \quad v=0 & & \text { in } \partial \Omega .
\end{array}
$$

Consider the following hypotheses:

$$
\begin{aligned}
& p, q \in] 1, \infty[\text { such that } \\
& \qquad \begin{array}{r}
1 / p>1 / 2-2 / N, \quad 1 / q>1 / 2-2 / N, \\
1 / p+1 / q>1-2 / N .
\end{array}
\end{aligned}
$$

(1.3) The function $H: \mathbb{R} \times \mathbb{R} \times \bar{\Omega} \rightarrow \mathbb{R}$ is of class $C^{2}$.

(1.4) There is a constant $\left.c_{1} \in\right] 0, \infty[$ such that for all $(\xi, \eta, x) \in \mathbb{R} \times \mathbb{R} \times \bar{\Omega}$,

$$
\begin{aligned}
& \left|\partial_{\xi} H(\xi, \eta, x)\right| \leq c_{1}\left(|\xi|^{p-1}+|\eta|^{(p-1) q / p}+1\right), \\
& \left|\partial_{\eta} H(\xi, \eta, x)\right| \leq c_{1}\left(|\eta|^{q-1}+|\xi|^{(q-1) p / q}+1\right) .
\end{aligned}
$$

(1.5) There are constants $\left.c_{2}, \delta \in\right] 0, \infty[$ such that for all $(\xi, \eta, x) \in \mathbb{R} \times$ $\mathbb{R} \times \bar{\Omega}$,

$$
\partial_{\xi} H(\xi, \eta, x) \xi-\partial_{\eta} H(\xi, \eta, x) \eta \geq-c_{2}+\delta\left(|\xi|^{p}+|\eta|^{q}\right) .
$$

Existence results for solutions of (1.1) under the above hypotheses were recently obtained by Angenent and van der Vorst in their important paper [1]. Functions $H$ satisfying Hypothesis (1.5) are called by these authors "-"-type Hamiltonians.

As shown in [1], the solutions of system (1.1) can be described as equilibria of a certain gradient-like ordinary differential equation

$$
\dot{z}=f(z)
$$

on an infinite-dimensional Hilbert space $E$ of functions $z=(u, v)$ (see Section 2). In general, this equation does not satisfy the uniqueness property of the Cauchy problem. Moreover, the equilibria of (1.6) are strongly indefinite, i.e. their stable and unstable manifolds are both infinite-dimensional. That is why the usual versions of Conley index theory are not applicable to this problem.

To remedy these difficulties the authors of [1] develop a version of MorseFloer homology for (1.6). This is a technically involved construction rendered even more difficult by the fact that, due to the lack of smoothness of the right-hand side of (1.6) on the Hilbert space $E$, one has to consider this equation on a certain Banach subspace of $E$.

It is the purpose of this paper to show that systems like (1.1) (and the corresponding ordinary differential equation (1.6)) can actually be investigated by Conley index methods. In fact, we study (1.6) directly in the 
Hilbert space $E$ by means of an elementary extension of the Conley index to certain classes of infinite-dimensional ordinary differential equations which do not necessarily have unique solutions.

This theory is presented in our previous paper [9]. Similarly to its less general predecessor defined in [6], it is based on the classical Conley index for flows in locally compact spaces together with Galerkin approximations: the index of a compact isolated invariant set relative to a given infinitedimensional equation is equal to the sequence of Conley indices relative to appropriate (finite-dimensional) Galerkin approximations.

The main result of the present paper is a Linearization Principle (Theorem 2.9) which states that whenever $z_{0}$ is an equilibrium of (1.6) which is hyperbolic (in the sense of Definition 2.8 below) then the Galerkin-type Conley index $h\left(\left\{z_{0}\right\}\right)$ of $\left\{z_{0}\right\}$ can be computed by formally linearizing (1.6) at $z_{0}$. This is nontrivial, since, in general, the map $f: E \rightarrow E$ occurring in (1.6) is not differentiable. It follows that there is an integer $\gamma$ such that $h\left(\left\{z_{0}\right\}\right)$ is equal to the sequence $\left(\Sigma^{l+\gamma}\right)_{l \geq l_{0}}$. It can be proved that the number $\gamma$ is actually equal to the renormalized Morse index of $z_{0}$ as defined in [1].

The proof of Theorem 2.9, although slightly involved, uses only properties of the Conley index together with a basic bootstrapping result from [1].

Using an a-priori-bound result established in [1] and the homotopy invariance of the Galerkin-type Conley index we show in Theorem 2.13 that the set of all full bounded orbits of (1.6) has Conley index equal to the sequence $\left(\Sigma^{l}\right)_{l \geq l_{1}}$.

Theorems 2.9 and 2.13 together with the variational structure of (1.6) imply that if $(u, v)=(0,0)$ is a solution of (1.1) which is a hyperbolic equilibrium of (1.6) with $\gamma \neq 0$, then system (1.1) must also have a nontrivial solution (cf. Theorem 2.16). This gives a simple Conley index based proof of one of the main results from [1].

An explicit formula for the Galerkin-type Conley index of a hyperbolic equilibrium of (2.9) appears in Theorem 2.6. The proof of this result is much simpler than the proof, using the spectral flow formula, of a corresponding result in [1] concerning the Morse-Floer index.

Using the elementary method developed in the present paper, together with some abstract results on Morse decompositions in the absence of uniqueness, obtained in the recent paper [3], one can also establish multiplicity results for solutions of strongly indefinite variational systems. In particular, we can prove a conjecture, made in [1], about the existence of multiple solutions of system (1.1) for even Hamiltonians. This will appear in [10].

The paper is organized as follows: In Section 2 we state and prove our main results, using some background material from [1]. For the reader's convenience, we provide proofs of the latter results in the Appendix. In par- 
ticular, we present a detailed proof of a basic bootstrapping result from [1] using an argument, based on discrete dynamical systems, which is of independent interest and which can be used in more general contexts.

We remark that the notation of this paper is similar to that of [5] and different from the notation used in [1]. In particular, we write $p$ (resp. $q$ ) where the authors of [1] write $p+1$ (resp. $q+1$ ).

Moreover, we denote by $\mathbb{N}$ (resp. $\mathbb{N}_{0}$ ) the set of positive (resp. nonnegative) integers.

We also remark that all linear spaces considered in this paper are over the real numbers (except that we pass to complexification when dealing with spectral theory).

2. Main results. In this section we state and prove the main results of this paper. We require a few basic results established in [1] and [5]. For clarity of exposition we include proofs of those results in the Appendix.

It is well known that the linear operator

$$
B: W^{2,2}(\Omega) \cap W_{0}^{1,2}(\Omega) \rightarrow L^{2}(\Omega), \quad u \mapsto-\Delta u,
$$

is positive self-adjoint and, consequently, sectorial in $X=L^{2}(\Omega)$. Thus $B$ generates a family $X^{\alpha}, \alpha \in[0, \infty[$, of fractional power spaces (cf. e.g. [8]). We write $A:=B^{1 / 2}$. Moreover, for $\alpha \in\left[0, \infty\left[\right.\right.$ let $E^{\alpha}:=X^{\alpha / 2}$ and $E^{-\alpha}:=E^{\alpha *}$ be the dual of $E^{\alpha}$. Note that for $\alpha \in[0, \infty[$ the formula

$$
\langle u, v\rangle_{\alpha}:=\left\langle A^{\alpha} u, A^{\alpha} v\right\rangle_{L^{2}}, \quad u, v \in E^{\alpha},
$$

defines a Hilbert product in $E^{\alpha}$ and $A^{\alpha}$ is an isometry between the Hilbert spaces $E^{\alpha}$ and $L^{2}(\Omega)$. Endow $E^{-\alpha}:=E^{\alpha *}$ with the dual product. We write

$$
A^{-\alpha}:=\left(A^{\alpha}\right)^{-1}: L^{2}(\Omega) \rightarrow E^{\alpha} .
$$

Whenever $\lambda>0$ and $B \phi=\lambda \phi$ then $A^{\beta} \phi=\lambda^{\beta / 2} \phi$ for every $\beta \in \mathbb{R}$.

It is well known that for every $\beta \in \mathbb{R}$ the operator $A^{\beta}$ can be uniquely extended to a map

$$
A^{\beta}: \bigcup_{\alpha \in \mathbb{R}} E^{\alpha} \rightarrow \bigcup_{\alpha \in \mathbb{R}} E^{\alpha}
$$

such that whenever $\alpha \in \mathbb{R}$ then $A^{\beta}\left(E^{\alpha}\right)=E^{\alpha-\beta}$ and $A_{\mid E^{\alpha}}^{\beta}: E^{\alpha} \rightarrow E^{\alpha-\beta}$ is an isometry. Moreover, $A^{0}$ is the identity on $\bigcup_{\alpha \in \mathbb{R}} E^{\alpha}$ and $A^{\beta} \circ A^{\gamma}=A^{\beta+\gamma}$ for all $\beta, \gamma \in \mathbb{R}$.

The following simple result is essentially known.

2.1. Proposition. Hypothesis (1.2) is equivalent to the following condition:

$$
p, q \in] 1, \infty[\text { and there are } s, t \in] 0, \infty[\text { such that } s+t=2 \text { and }
$$

$$
1 / p>1 / 2-s / N, \quad 1 / q>1 / 2-t / N \text {. }
$$


Proof. Assume (2.1). Then $s, t \in[0,2]$ and so $1 / p>1 / 2-s / N \geq 1 / 2-$ $2 / N$. Similarly, $1 / q>1 / 2-2 / N$. Consequently, $1 / p+1 / q>1 / 2-s / N+$ $1 / 2-t / N=1-2 / N$ and so Hypothesis (1.2) is satisfied.

Now assume (1.2). Then it follows from the third inequality in (1.2) that $1 / 2-1 / p<2 / N-1 / 2+1 / q$, while the first and second inequalities imply that the set

$$
I:=] 1 / 2-1 / p, 2 / N-1 / 2+1 / q[\cap] 0,2 / N[
$$

is nonempty. Thus we can choose $s^{\prime} \in I$ and set $s:=N s^{\prime}$ and $t:=2-s$. With this choice of $s$ and $t$ condition (2.1) is satisfied.

From now on we assume Hypotheses (1.2)-(1.5) and let $s$ and $t$ be as in Proposition 2.1.

Define the product Hilbert space $E:=E^{s} \times E^{t}$ with the Hilbert product

$$
\left\langle z, z^{\prime}\right\rangle:=\left\langle u, u^{\prime}\right\rangle_{s}+\left\langle v, v^{\prime}\right\rangle_{t}, \quad z=(u, v), z^{\prime}=\left(u^{\prime}, v^{\prime}\right) \in E .
$$

We write $|\cdot|_{E}$ to denote the Hilbert space norm on $E$. Moreover, given $z=(u, v)$ we write $\bar{z}:=(u,-v)$. Finally, if $G: E \rightarrow \mathbb{R}$ is a $C^{1}$-function, then we denote by $\nabla G$ the gradient of $G$ with respect to the scalar product $\langle\cdot, \cdot\rangle$.

Note that, by a well known result from interpolation theory (cf. [8]),

$$
\text { whenever } r \in[1, \infty[\text { and } \sigma \in] 0, \infty[\text { are such that }
$$

$$
1 / r>1 / 2-\sigma / N
$$

then the imbedding $E^{\sigma} \subset L^{r}(\Omega)$ is well defined and compact.

Now set

$$
L(u, v):=\left(A^{-s} A^{t} v, A^{-t} A^{s} u\right), \quad(u, v) \in E .
$$

This clearly defines a linear operator $L: E \rightarrow E$. The following well known result collects the basic properties of $L$ :

2.2. Proposition (cf. Proposition 1.1 of [5]). 1. The linear operator $L: E \rightarrow E$ is an $(E,\langle\cdot, \cdot\rangle)$-symmetric isometry such that $\langle L z, \bar{z}\rangle=0$ for all $z \in E$.

2. L has two eigenvalues, $\lambda=-1$ and $\lambda=1$, with the corresponding eigenspaces $E_{-1}$ and $E_{1}$ given as

$$
E_{-1}=\left\{\left(u,-A^{-t} A^{s} u\right) \mid u \in E^{s}\right\}, \quad E_{1}=\left\{\left(u, A^{-t} A^{s} u\right) \mid u \in E^{s}\right\} .
$$

The spaces $E_{-1}$ and $E_{1}$ are $E$-orthogonal complements to each other, and so, in particular, $E=E_{-1} \oplus E_{1}$. The maps $Q_{-1}: E \rightarrow E$ and $Q_{1}: E \rightarrow E$ given as

$$
\begin{aligned}
Q_{-1}(u, v) & =\frac{1}{2}\left(u-A^{-s} A^{t} v, v-A^{-t} A^{s} u\right), & & (u, v) \in E \\
Q_{1}(u, v) & =\frac{1}{2}\left(u+A^{-s} A^{t} v, v+A^{-t} A^{s} u\right), & & (u, v) \in E
\end{aligned}
$$


are orthogonal (hence $(E,\langle\cdot, \cdot\rangle)$-symmetric) projectors onto $E_{-1}$ and $E_{1}$ along the above direct sum decomposition.

3. Let $\left(\lambda_{k}\right)_{k \in \mathbb{N}}$ be the repeated nondecreasing sequence of eigenvalues of $B$ and $\left(\phi_{k}\right)_{k \in \mathbb{N}}$ be a corresponding $L^{2}$-orthogonal sequence of eigenvectors such that $\left|\phi_{k}\right|_{L^{2}}^{2}=1 / 2$ for every $k \in \mathbb{N}$. For every $k \in \mathbb{N}$ let

$$
\chi_{k}=\left(A^{-s} \phi_{k}, A^{-t} \phi_{k}\right) \text {. }
$$

Then $\left(\chi_{k}\right)_{k \in \mathbb{N}}$ is an E-orthonormal basis of $E_{1}$, while $\left(\bar{\chi}_{k}\right)_{k \in \mathbb{N}}$ is an Eorthonormal basis of $E_{-1}$.

For every $l \in \mathbb{N}$ define the map $P^{l}: E \rightarrow E$ by

$$
P^{l}(u, v)=\sum_{k=1}^{l}\left\langle Q_{-1}(u, v), \bar{\chi}_{k}\right\rangle \bar{\chi}_{k}+\sum_{k=1}^{l}\left\langle Q_{1}(u, v), \chi_{k}\right\rangle \chi_{k}, \quad(u, v) \in E .
$$

The maps $P^{l}$ are E-orthogonal projection operators and $P^{l} \rightarrow \operatorname{Id}_{E}$ as $l \rightarrow \infty$, uniformly on compact subsets of $E$. The spaces $P^{l}(E)$ are finite-dimensional (hence closed) and L-invariant.

4. Let $K: E \rightarrow E$ be linear, $(E,\langle\cdot, \cdot\rangle)$-symmetric and compact. Then the spectrum of the operator $L+K$ consists of $-1,1$ and the (countable) set of real eigenvalues of $L+K$. The following conditions are equivalent:

$$
\begin{gathered}
z(\cdot) \equiv 0 \text { is the only full bounded solution of the equation } \\
\dot{z}=(L+K) z ;
\end{gathered}
$$

$$
L+K \text { is hyperbolic, i.e. } \mu=0 \text { is not an eigenvalue of } L+K \text {. }
$$

Proof. The proof follows by straightforward calculations, using standard results from spectral theory. Easy details are left to the reader.

Now note that, in view of (1.4), for $u \in L^{p}(\Omega)$ and $v \in L^{q}(\Omega)$ the function $\partial_{\xi} H(u(\cdot), v(\cdot), \cdot)$ lies in $L^{p /(p-1)}(\Omega)$ so we may regard $\partial_{\xi} H(u(\cdot), v(\cdot), \cdot)$ as an element of the dual space of $L^{p}(\Omega)$ and hence, by $(2.2)$, we can regard $\partial_{\xi} H(u(\cdot), v(\cdot), \cdot)$ as an element of $E^{-s}$. Thus $A^{-2 s} \partial_{\xi} H(u(\cdot), v(\cdot), \cdot)$ is a well defined element of $E^{s}$. Similarly, we may regard the function $\partial_{\eta} H(u(\cdot), v(\cdot), \cdot)$ as an element of $E^{-t}$ so $A^{-2 t} \partial_{\eta} H(u(\cdot), v(\cdot), \cdot)$ is a well defined element of $E^{t}$. We thus obtain a well defined map

$$
G: E \rightarrow E, \quad(u, v) \mapsto\left(G_{1}(u, v), G_{2}(u, v)\right),
$$

where

$$
\begin{aligned}
& G_{1}(u, v)=A^{-2 s} \partial_{\xi} H(u(\cdot), v(\cdot), \cdot), \\
& G_{2}(u, v)=A^{-2 t} \partial_{\eta} H(u(\cdot), v(\cdot), \cdot) .
\end{aligned}
$$

For $\theta \in[0,1]$ let $K_{\theta}$ be the map

$$
K_{\theta}: E \rightarrow E, \quad z \mapsto-\theta G(z),
$$

and $f_{K_{\theta}}=L+K_{\theta}$. 
We have the following result, whose proof is given in the Appendix.

2.3. Proposition. For every $\theta \in[0,1]$ the map $K_{\theta}: E \rightarrow E$ is continuous and whenever $N \subset E$ is bounded, then the set $\bigcup_{\theta \in[0,1]} K_{\theta}(N)$ is relatively compact in E. Moreover,

$$
K_{\theta}=-\theta \nabla \psi, \quad \theta \in[0,1],
$$

where

$$
\psi: E \rightarrow \mathbb{R}, \quad(u, v) \mapsto \int_{\Omega} H(u(x), v(x), x) d x .
$$

Since $L$ is $E$-symmetric, we thus obtain

$$
f_{\theta}:=f_{K_{\theta}}=\nabla \Phi_{\theta}, \quad \theta \in[0,1],
$$

where

$$
\Phi_{\theta}: E \rightarrow \mathbb{R}, \quad z \mapsto \frac{1}{2}\langle L z, z\rangle-\theta \psi(z) .
$$

We need the following important bootstrapping result:

2.4. Proposition (cf. Section 3.4 of [1]). Let $I$ be an arbitrary set and for every $i \in I$ let $\pi_{i}, \varrho_{i}: \mathbb{R} \times \mathbb{R} \times \bar{\Omega} \rightarrow \mathbb{R}$ be $C^{1}$-functions. Assume that there is a constant $c \in[0, \infty[$ such that for all $i \in I$ and all $(\xi, \eta, x) \in \mathbb{R} \times \mathbb{R} \times \bar{\Omega}$,

$$
\begin{aligned}
& \left|\pi_{i}(\xi, \eta, x)\right| \leq c\left(|\xi|^{p-1}+|\eta|^{(p-1) q / p}+|\xi|+|\eta|+1\right), \\
& \left|\varrho_{i}(\xi, \eta, x)\right| \leq c\left(|\eta|^{q-1}+|\xi|^{(q-1) p / q}+|\xi|+|\eta|+1\right) .
\end{aligned}
$$

Let $Z$ be the set of all $z=(u, v) \in E$ for which there is an $i \in I$ such that

$$
L z+\left(A^{-2 s} \pi_{i}(u(\cdot), v(\cdot), \cdot), A^{-2 t} \varrho_{i}(u(\cdot), v(\cdot), \cdot)\right)=0 .
$$

Then there is a $\beta \in] 0,1\left[\right.$ and for every $c^{\prime} \in\left[0, \infty\left[\right.\right.$ there is a $c^{\prime \prime} \in[0, \infty[$ such that whenever $z \in Z$ is such that $|z|_{E} \leq c^{\prime}$, then $z \in C_{0}^{2, \beta}(\bar{\Omega}) \times C_{0}^{2, \beta}(\bar{\Omega})$ and $|z|_{C^{2, \beta} \times C^{2, \beta}} \leq c^{\prime \prime}$.

The proof of Proposition 2.4 is given in the Appendix.

As a simple corollary of Proposition 2.4 we obtain the following result:

2.5. Proposition. $(u, v)$ is a classical solution of (1.1) if and only if $(u, v) \in E$ and $f_{K_{1}}(u, v)=0$.

Thus (2.7) and Proposition 2.5 show that the study of solutions of system (1.1) is reduced to the study of the equilibria of the gradient-like ordinary differential equation

$$
\dot{z}=f_{K_{1}}(z)
$$

on $E$. Therefore one may try to use ideas from Conley index theory ([4] or [11]) to search for solutions of (1.1). However, since we do not impose any growth restrictions on the second partial derivatives of $H$ with respect to the variables $(\xi, \eta)$, the map $f_{K_{1}}: E \rightarrow E$ is not, in general, differentiable 
nor even locally Lipschitzian, and the Cauchy problem for (2.9) may have nonunique solutions.

For this reason, in the present paper, we use a version of Conley index theory developed in [9], which does not require uniqueness of the Cauchy problem.

We assume that the reader is familiar with the paper [9] and its terminology. In what follows we will use the letter $N$ to denote both the space dimension of system (1.1) and various subsets of $E$. This should not lead to confusion.

Proposition 2.2 implies that Hypotheses 4.2, 4.6 and 4.8 of [9] are satisfied.

Suppose now that $K: E \rightarrow E$ is continuous and $N \subset E$ is bounded, closed and such that $K(N)$ is relatively compact in $E$. Moreover, assume that $N$ is an isolating neighborhood with respect to the ordinary differential equation

$$
\dot{z}=f_{K}(z)
$$

on $E$, where

$$
f_{K}: E \rightarrow E, \quad z \mapsto L z+K(z) .
$$

Then it follows from Proposition 4.7 of [9] that there is a smallest $l_{0}(K, N) \in$ $\mathbb{N}$ such that for every $l \geq l_{0}(K, N)$ the set $N \cap P^{l}(E)$ is an isolating neighborhood with respect to the lth Galerkin approximation $f_{P^{l} \circ K}^{l}$ of $f$ defined by the formula

$$
f_{P^{l} \circ K}^{l}: P^{l}(E) \rightarrow P^{l}(E), \quad z \mapsto L z+P^{l} K(z) .
$$

By Definition 4.9 of [9] the Conley index $h\left(f_{K}, N\right)$ is defined as

$$
h\left(f_{K}, N\right)=\left(h\left(f_{K}, N\right)_{l}\right)_{l \geq l_{0}(K, N)}
$$

where $h\left(f_{K}, N\right)_{l}=h\left(f_{P^{l} \circ K}, N \cap P^{l}(E)\right)$ is the finite-dimensional Conley index defined in [9], Section 3. Note that uniqueness of the Cauchy problem is not assumed. Now Proposition 2.2 implies that Hypothesis 4.16 of [9] with $i_{l} \equiv 1$ is satisfied. Thus, by Proposition 4.18 of [9] we have

$$
h\left(f_{K}, N\right)_{l+1}=\Sigma^{1} \wedge h\left(f_{K}, N\right)_{l}, \quad l \geq l_{0}(K, N) .
$$

It is proved in [9] that $h\left(f_{K}, N\right)$ depends only on the isolated invariant set isolated by $N$. More precisely, if $N$ and $N^{\prime}$ are both bounded closed in $E$ with $K(N)$ and $K\left(N^{\prime}\right)$ relatively compact in $E$ and if $N$ and $N^{\prime}$ both isolate the same invariant set $S$, then

$$
h\left(f_{K}, N\right)_{l}=h\left(f_{K}, N^{\prime}\right)_{l} \quad \text { for all } l \in \mathbb{N} \text { large enough. }
$$

We can therefore simply write $h\left(f_{K}, S\right):=h\left(f_{K}, N\right)$ and speak of the Conley index of $\left(f_{K}, S\right)$. This will not lead to confusion. 
The following result is a Conley index analogue of Lemma 30 of [1].

2.6. Theorem. Let $a, b, c \in \mathbb{R}$. Define the map

$$
K=K_{\text {lin }}: E \rightarrow E, \quad(u, v) \mapsto\left(A^{-2 s}(-a u+c v), A^{-2 t}(c u-b v)\right) .
$$

Then:

1. The map $K$ is linear, $(E,\langle\cdot, \cdot\rangle)$-symmetric and compact.

2. The linear operator $f_{K}:=L+K$ is hyperbolic if and only if

$$
\left(\lambda_{k}+c\right)^{2}-b a \neq 0 \quad \text { for all } k \in \mathbb{N} .
$$

3. Suppose that (2.12) is satisfied and let $r \in \mathbb{N}_{0}$ be the number of $k \in \mathbb{N}$ such that $\left(\lambda_{k}+c\right)^{2}-b a<0$. Then $\{0\}$ is an isolated invariant set of $f_{K}$ and for every bounded isolating neighborhood $N$ of $\{0\}$ the index $h\left(f_{K},\{0\}\right)=$ $h\left(f_{K}, N\right)$ is given by the formula

$$
h\left(f_{K},\{0\}\right)=\left(h\left(f_{K}, N\right)_{l}\right)_{l \geq r}
$$

where, for $l \geq r$,

$$
h\left(f_{K}, N\right)_{l}= \begin{cases}\Sigma^{l} & \text { if } a b \leq 0 ; \\ \Sigma^{l-r} & \text { if } a, b<0 ; \\ \Sigma^{l+r} & \text { if } a, b>0 .\end{cases}
$$

Proof. Part 1 is clear. In particular, the map $K: E \rightarrow E$ is compact, since, by (2.2), the inclusion $E \subset L^{p}(\Omega) \times L^{q}(\Omega)$ is compact.

It is easily seen that for every $k \in \mathbb{N}$ the operator $f_{K}$ maps the twodimensional space $F_{k}$ spanned by $\left\{\chi_{k}, \bar{\chi}_{k}\right\}$ into itself. Let $f_{K, k}: F_{k} \rightarrow F_{k}$ be the restriction of $f_{K}$ to $F_{k}$. The matrix representation $M_{k}=\left(a_{k, i j}\right)_{i, j=1,2}$ of $f_{K, k}$ in the basis $\chi_{k}, \bar{\chi}_{k}$ is given by

$$
\begin{aligned}
& 2 a_{k, 11}=2-a \lambda_{k}^{-s}+2 c \lambda_{k}^{-1}-b \lambda_{k}^{-t}, \\
& 2 a_{k, 12}=-a \lambda_{k}^{-s}+b \lambda_{k}^{-t}, \\
& 2 a_{k, 22}=-2-a \lambda_{k}^{-s}-2 c \lambda_{k}^{-1}-b \lambda_{k}^{-t},
\end{aligned}
$$

and $a_{k, 21}=a_{k, 12}$. The eigenvalues $\mu_{k, 1}, \mu_{k, 2}$ of $M_{k}$ with $\mu_{k, 1} \leq \mu_{k, 2}$ are given by the formulas

$$
\begin{aligned}
& 2 \mu_{k, 1}=a \lambda_{k}^{-s}+b \lambda_{k}^{-t}-\left[\left(b \lambda_{k}^{-t}-a \lambda_{k}^{-s}\right)^{2}+4\left(1+c \lambda_{k}^{-1}\right)^{2}\right]^{1 / 2}, \\
& 2 \mu_{k, 2}=a \lambda_{k}^{-s}+b \lambda_{k}^{-t}+\left[\left(b \lambda_{k}^{-t}-a \lambda_{k}^{-s}\right)^{2}+4\left(1+c \lambda_{k}^{-1}\right)^{2}\right]^{1 / 2} .
\end{aligned}
$$

Thus

$$
\begin{aligned}
& 2 \mu_{k, 1}=a \lambda_{k}^{-s}+b \lambda_{k}^{-t}-\left[\left(a \lambda_{k}^{-s}+b \lambda_{k}^{-t}\right)^{2}+4 \lambda_{k}^{-2}\left(\left(\lambda_{k}+c\right)^{2}-b a\right)\right]^{1 / 2}, \\
& 2 \mu_{k, 2}=a \lambda_{k}^{-s}+b \lambda_{k}^{-t}+\left[\left(a \lambda_{k}^{-s}+b \lambda_{k}^{-t}\right)^{2}+4 \lambda_{k}^{-2}\left(\left(\lambda_{k}+c\right)^{2}-b a\right)\right]^{1 / 2} .
\end{aligned}
$$


This implies that:

$$
\begin{aligned}
& \left(\lambda_{k}+c\right)^{2}-b a=0 \text { if and only if } \mu_{k, 1}=0 \text { or } \mu_{k, 2}=0 ; \\
& \text { if }\left(\lambda_{k}+c\right)^{2}-b a>0 \text { then } \mu_{k, 1}<0<\mu_{k, 2} ; \\
& \text { if }\left(\lambda_{k}+c\right)^{2}-b a<0 \text { and } a, b>0 \text { then } 0<\mu_{k, 1} \leq \mu_{k, 2} ; \\
& \text { if }\left(\lambda_{k}+c\right)^{2}-b a<0 \text { and } a, b<0 \text { then } \mu_{k, 1} \leq \mu_{k, 2}<0 .
\end{aligned}
$$

Since $E$ is the direct sum of the spaces $F_{k}, k \in \mathbb{N}$, it follows that a number $\mu$ is an eigenvalue of $f_{K}$ if and only if $\mu$ is an eigenvalue of $f_{K, k}$ for some $k \in \mathbb{N}$. Thus (2.13) together with Proposition 2.2 implies part 2 of the proposition.

Now assume (2.12). Consequently, $\{0\}$ is an isolated invariant set of $f_{K}$. Let $N$ be an arbitrary bounded isolated neighborhood of $\{0\}$ and let $l \in \mathbb{N}$ with $l \geq r$. The space $P^{l}(E)$ is the direct sum of the spaces $F_{k}, k=1, \ldots, l$. If $a b \leq 0$, then $\left(\lambda_{k}+c\right)^{2}-b a \geq 0$ for all $k=1, \ldots, l$, so $r=0$ and, by (2.14), the linear map $f_{P^{l} \circ K}^{l}$ has exactly $l$ positive and $l$ negative eigenvalues. Thus $h\left(f_{K}, N\right)_{l}=\Sigma^{l}$. If $a, b<0$, then by (2.14) and (2.16) we see that the linear map $f_{P^{l} \circ K}^{l}$ has exactly $l-r$ positive and $l+r$ negative eigenvalues. Thus $h\left(f_{K}, N\right)_{l}=\Sigma^{l-r}$. Analogously, if $a, b>0$, then $h\left(f_{K}, N\right)_{l}=\Sigma^{l+r}$. This proves part 3 of the proposition.

2.7. Remark. Note the completely elementary character of the proof of Theorem 2.6. In particular, our proof does not require the spectral flow formula used in [1] and [2].

We now make the following useful definition.

2.8. Definition. Suppose $z_{0}=\left(u_{0}, v_{0}\right) \in E$ is an equilibrium of $(2.9)$, i.e. $f_{K_{1}}\left(z_{0}\right)=0$. Define the linear map $K_{\operatorname{lin}, z_{0}}: E \rightarrow E$ by

$$
K_{\operatorname{lin}, z_{0}}(u, v)=\left(A^{-2 s}(-a(\cdot) u+c(\cdot) v), A^{-2 t}(c(\cdot) u-b(\cdot) v)\right) .
$$

Here, the continuous functions $a, b, c: \bar{\Omega} \rightarrow \mathbb{R}$ are defined, for $x \in \bar{\Omega}$, by

$$
\begin{gathered}
a(x)=\partial_{\xi \xi} H\left(z_{0}(x), x\right), \quad b(x)=\partial_{\eta \eta} H\left(z_{0}(x), x\right), \\
-c(x)=\partial_{\xi \eta} H\left(z_{0}(x), x\right)=\partial_{\eta \xi} H\left(z_{0}(x), x\right) .
\end{gathered}
$$

We call the equilibrium $z_{0}$ hyperbolic if the linear operator $L+K_{\operatorname{lin}, z_{0}}: E \rightarrow$ $E$ is hyperbolic.

The following Linearization Principle is one of the main contributions of this paper. It shows that one can compute the Conley index of a hyperbolic equilibrium point $z_{0}$ of $(2.9)$ by formally linearizing the equation at $z_{0}$. The result is nontrivial since, in general, the nonlinearity $f_{K_{1}}: E \rightarrow E$ is not differentiable at $z_{0}$.

2.9. Theorem. Let $z_{0}=\left(u_{0}, v_{0}\right) \in E$ be a hyperbolic equilibrium of (2.9). Set $K_{\operatorname{lin}}:=K_{\operatorname{lin}, z_{0}}$. Then $\left\{z_{0}\right\}$ is an isolated invariant set for $f_{K_{1}}$ and

$$
h\left(f_{K_{1}},\left\{z_{0}\right\}\right)_{l}=h\left(f_{K_{\text {lin }}},\{0\}\right)_{l} \quad \text { for all } l \in \mathbb{N} \text { large enough } .
$$


Proof. As mentioned before, this result would be standard if the map $f_{K_{1}}$ were differentiable at $z_{0}$ with derivative $f_{K_{\text {lin }}}$. However, in general, this is not the case and so the reasoning is somewhat more involved.

Define the map $K_{\text {aff }}: E \rightarrow E$ by

$$
K_{\text {aff }}(z)=K_{1}\left(z_{0}\right)+K_{\operatorname{lin}}\left(z-z_{0}\right)=-L z_{0}+K_{\operatorname{lin}}\left(z-z_{0}\right), \quad z \in E .
$$

Here we use the fact that $L z_{0}+K_{1}\left(z_{0}\right)=0$. We first claim that

there is a closed bounded set $N \subset E$ such that for every $\kappa \in[0,1]$ the set $N$ is an isolating neighborhood of $\left\{z_{0}\right\}$ with respect to $L+\kappa K_{1}+(1-\kappa) K_{\text {aff }}$.

To prove this claim note that

$$
L+\kappa K_{1}+(1-\kappa) K_{\mathrm{aff}}=\nabla \mathcal{L}_{\kappa}
$$

where $\mathcal{L}: E \rightarrow \mathbb{R}$ is defined by

$$
\mathcal{L}(z)=\kappa\left(\frac{1}{2}\langle L z, z\rangle-\psi(z)\right)+\frac{1}{2}(1-\kappa)\left\langle\left(L+K_{\text {lin }}\right)\left(z-z_{0}\right), z-z_{0}\right\rangle
$$

for $z \in E$. Thus, in the terminology of [9], for every $\kappa \in[0,1]$ the pair $\left(L+\kappa K_{1}+(1-\kappa) K_{\mathrm{aff}}, E\right)$ is gradient-like with respect to $-\mathcal{L}_{\kappa}$. Therefore, if (2.18) is not true, then there are sequences $\left(\kappa_{n}\right)_{n \in \mathbb{N}}$ in $[0,1]$ and $\left(z_{n}\right)_{n \in \mathbb{N}}$ in $E$ with $z_{n} \rightarrow z_{0}$ in $E$ and such that for every $n \in \mathbb{N}, z_{n} \neq z_{0}$ and

$$
L z_{n}+\kappa_{n} K_{1}\left(z_{n}\right)+\left(1-\kappa_{n}\right) K_{\text {aff }} z_{n}=0 .
$$

Now write $\zeta_{n}=z_{n}-z_{0}$ for $n \in \mathbb{N}$. Then

$$
\begin{aligned}
L z_{n}+\kappa_{n} K_{1}\left(z_{n}\right) & +\left(1-\kappa_{n}\right) K_{\mathrm{aff}} z_{n} \\
& =\left(L+K_{\operatorname{lin}}\right) \zeta_{n}+\kappa_{n}\left(K_{1}\left(\zeta_{n}+z_{0}\right)-K_{1}\left(z_{0}\right)-K_{\operatorname{lin}} \zeta_{n}\right)
\end{aligned}
$$

so we obtain

$$
\left(L+K_{\operatorname{lin}}\right) \zeta_{n}+\kappa_{n}\left(K_{1}\left(\zeta_{n}+z_{0}\right)-K_{1}\left(z_{0}\right)-K_{\operatorname{lin}} \zeta_{n}\right)=0 .
$$

For $n \in \mathbb{N}$ and $x \in \bar{\Omega}$ define

$$
\begin{aligned}
\sigma_{n}(x) & =\int_{0}^{1}\left(\partial_{\xi \xi} H\left((1-r) z_{0}(x)+r z_{n}(x), x\right)-\partial_{\xi \xi} H\left(z_{0}(x), x\right)\right) d r, \\
\tau_{n}(x) & =\int_{0}^{1}\left(\partial_{\eta \eta} H\left((1-r) z_{0}(x)+r z_{n}(x), x\right)-\partial_{\eta \eta} H\left(z_{0}(x), x\right)\right) d r, \\
\omega_{n}(x) & =\int_{0}^{1}\left(\partial_{\xi \eta} H\left((1-r) z_{0}(x)+r z_{n}(x), x\right)-\partial_{\xi \eta} H\left(z_{0}(x), x\right)\right) d r .
\end{aligned}
$$

Notice that (2.19) can be written in the form

$$
L \zeta_{n}+\left(A^{-2 s} \pi_{n}\left(\widetilde{u}_{n}(\cdot), \widetilde{v}_{n}(\cdot), \cdot\right), A^{-2 t} \varrho_{n}\left(\widetilde{u}_{n}(\cdot), \widetilde{v}_{n}(\cdot), \cdot\right)\right)=0
$$


where $\left(\widetilde{u}_{n}, \widetilde{v}_{n}\right)=\zeta_{n}, n \in \mathbb{N}$, while $\pi_{n}, \varrho_{n}, n \in I:=\mathbb{N}$, are functions satisfying the assumptions of Proposition 2.4. (Here we use the fact that $z_{0} \in C^{0}(\bar{\Omega}) \times$ $C^{0}(\bar{\Omega})$ by Proposition 2.5 so $z_{0}$ is bounded.)

Since $\zeta_{n} \rightarrow 0$ in $E$, we conclude from (2.19) and Proposition 2.4 that $z_{n}-z_{0}=\zeta_{n} \rightarrow 0$ in $C^{0}(\bar{\Omega}) \times C^{0}(\bar{\Omega})$.

Hence the maps $\sigma_{n}, \tau_{n}, \omega_{n}: \bar{\Omega} \rightarrow \mathbb{R}$ are continuous and converge to zero in $C^{0}(\bar{\Omega})$. For $n \in \mathbb{N}$ let $\alpha_{n}:=\left|\zeta_{n}\right|_{C^{0} \times C^{0}}$ and $w_{n}=\left(u_{n}, v_{n}\right):=\zeta_{n} / \alpha_{n}$. Using (2.19) and the mean-value theorem we see that

$$
L w_{n}+K_{\operatorname{lin}} w_{n}=\kappa_{n}\left(A^{-2 s}\left(\sigma_{n} u_{n}+\omega_{n} v_{n}\right), A^{-2 t}\left(\omega_{n} u_{n}+\tau_{n} v_{n}\right)\right) .
$$

Since the operator $L+K_{\operatorname{lin}}$ is hyperbolic, its inverse $\left(L+K_{\operatorname{lin}}\right)^{-1}: E \rightarrow E$ exists and is continuous. Our hypotheses also imply that $\sigma_{n} u_{n}+\omega_{n} v_{n}$ and $\omega_{n} u_{n}+\tau_{n} v_{n}$ converge to zero in $C^{0}(\bar{\Omega})$, hence in $L^{2}(\Omega)$. Thus

$$
\kappa_{n}\left(A^{-2 s}\left(\sigma_{n} u_{n}+\omega_{n} v_{n}\right), A^{-2 t}\left(\omega_{n} u_{n}+\tau_{n} v_{n}\right)\right) \rightarrow 0
$$

in $E$ and so (2.20) implies that $w_{n} \rightarrow 0$ in $E$. Using (2.20) and applying Proposition 2.4 again we see that $w_{n} \rightarrow 0$ in $C^{0}(\bar{\Omega}) \times C^{0}(\bar{\Omega})$. However, this is a contradiction since $\left|w_{n}\right|_{C^{0} \times C^{0}} \equiv 1$. Hence (2.18) holds.

Now (2.18) and the continuation invariance property of the Conley index (Proposition 4.13 of [9]) imply that

$$
h\left(f_{K_{1}}, N\right)_{l}=h\left(f_{K_{\text {aff }}}, N\right)_{l} \quad \text { for all } l \text { large enough. }
$$

For $n \in \mathbb{N}$ define

$$
K_{(n)}: E \rightarrow E, \quad z \mapsto K_{\operatorname{lin}}\left(z-P^{n} z_{0}\right)-L P^{n} z_{0} .
$$

Then $K_{(n)}: E \rightarrow E$ for every $n \in \mathbb{N}$ and since $K_{1}\left(z_{0}\right)=-L z_{0}$ it is clear that $K_{(n)} \rightarrow K_{\text {aff }}$ as $n \rightarrow \infty$, uniformly on compact subsets of $N$. Moreover, the sets $K_{\text {aff }}(N)$ and $\bigcup_{n \in \mathbb{N}} K_{(n)}(N)$ are relatively compact in $E$. Thus the assumptions of Proposition 4.7 of [9] are satisfied, so there are $l_{0}, n_{0} \in \mathbb{N}$ such that

$$
h\left(f_{P^{l} \circ K_{\mathrm{aff}}}^{l}, N \cap P^{l}(E)\right)=h\left(f_{P^{l} \circ K_{(n)}}^{l}, N \cap P^{l}(E)\right), \quad l \geq l_{0}, n \geq n_{0} .
$$

Thus

$$
\begin{aligned}
h\left(f_{K_{\mathrm{aff}}}, N\right)_{l} & =h\left(f_{P^{l} \circ K_{\mathrm{aff}}}^{l}, N \cap P^{l}(E)\right) \\
& =h\left(f_{P^{l} \circ K_{(l)}}^{l}, N \cap P^{l}(E)\right), \quad l \geq \max \left(l_{0}, n_{0}\right) .
\end{aligned}
$$

Now $f_{P^{l} \circ K_{(l)}}^{l}$ defines the ODE

$$
\dot{z}=L\left(z-P^{l} z_{0}\right)+P^{l} K_{\operatorname{lin}}\left(z-P^{l} z_{0}\right)
$$

on $P^{l}(E)$ which is conjugate, via the homeomorphism $P^{l}(E) \rightarrow P^{l}(E)$, $\zeta \mapsto \zeta+P^{l} z_{0}$, to the ODE

$$
\dot{\zeta}=L \zeta+P^{l} K_{\operatorname{lin}} \zeta .
$$


Since the classical Conley index is invariant under flow conjugation, we obtain

$$
h\left(f_{P^{l} \circ K_{(l)}}^{l}, N \cap P^{l}(E)\right)=h\left(f_{P^{l} \circ K_{\operatorname{lin}}}^{l},\left(N-P^{l} z_{0}\right) \cap P^{l}(E)\right)
$$

for all $l$ large enough. Here, of course,

$$
N-P^{l} z_{0}=\left\{z-P^{l} z_{0} \mid z \in N\right\} .
$$

It is easy to see that for all $l$ large enough the linear map $f_{P^{l} \circ K_{\operatorname{lin}}}^{l}: P^{l}(E) \rightarrow$ $P^{l}(E)$ is hyperbolic. In fact, let $\widetilde{N}$ be an arbitrary bounded isolating neighborhood of $\{0\}$ relative to $f_{K_{\text {lin }}}$. Then, by Proposition 4.7 of [9], for all $l$ large enough the set $\widetilde{N} \cap P^{l}(E)$ is an isolating neighborhood relative to $f_{P^{l} \circ K_{\text {lin }}}^{l}$. In particular, the map $f_{P^{l} \circ K_{\text {lin }}}^{l}$ must be hyperbolic, since otherwise no bounded set would be an isolating neighborhood. Hence, for all $l$ large enough, $P^{l} z_{0} \in \operatorname{Int} N$ and $\left(N-P^{l} z_{0}\right) \cap P^{l}(E)$ is an isolating neighborhood of $\{0\}$ relative to $f_{P^{l} \circ K_{\text {lin }}}^{l}$ so

$$
h\left(f_{P^{l} \circ K_{\operatorname{lin}}}^{l},\left(N-P^{l} z_{0}\right) \cap P^{l}(E)\right)=h\left(f_{P^{l} \circ K_{\operatorname{lin}}}^{l},\{0\}\right)=h\left(f_{K_{\operatorname{lin}}},\{0\}\right)_{l} .
$$

Now (2.21)-(2.24) imply (2.17).

2.10. Corollary. Under the assumptions of Theorem 2.9 there is an integer $\gamma$ such that

$$
h\left(f_{K_{1}},\left\{z_{0}\right\}\right)_{l}=h\left(f_{K_{\mathrm{lin}}},\{0\}\right)_{l}=\Sigma^{l+\gamma} \quad \text { for all } l \in \mathbb{N} \text { large enough } .
$$

Proof. Let $\widetilde{N}$ be an arbitrary bounded neighborhood of 0 . By the arguments used in the proof of Theorem 2.9 there is an $l_{1} \in \mathbb{N}$ such that the linear map $f_{P^{l} \circ K_{\text {lin }}}^{l}: P^{l}(E) \rightarrow P^{l}(E)$ is hyperbolic for all $l \geq l_{1}$. Thus there is an integer $\gamma \in\left[-l_{1}, l_{1}\right]$ such that

$$
h\left(f_{K_{\text {lin }}}, \widetilde{N}\right)_{l_{1}}=\Sigma^{l_{1}+\gamma} .
$$

Formulas (2.11) and (2.25) immediately imply that

$$
h\left(f_{K_{\operatorname{lin}}}, \tilde{N}\right)_{l}=\Sigma^{l+\gamma}, \quad l \geq l_{1} .
$$

This proves the corollary.

2.11. Remark. It can be proved (see [10]) that the number $\gamma$ is equal to the renormalized Morse index $\mu_{0}\left(z_{0}\right)$ of $z_{0}$, as introduced in Definition 17 of $[1]$.

The following result, established in [1], shows in particular that the set of all full bounded orbits of (2.9) is bounded, hence compact. This result depends in an essential way on the assumption that $H$ is a "-"-type Hamiltonian.

2.12. Proposition (cf. Lemma 4 of [1]). There is an $M \in] 0, \infty[$ such that whenever $\theta \in[0,1]$ and $z: \mathbb{R} \rightarrow E$ is a (full) bounded solution of 
$f_{K_{\theta}}=\nabla \Phi_{\theta}$ then

$$
\sup _{t \in \mathbb{R}}|z(t)|_{E} \leq M .
$$

For the reader's convenience, we give a proof of Proposition 2.12 in the Appendix.

We can now compute the Conley index of the set of all full bounded orbits of (2.9), obtaining the following Conley-index analogue of Theorem 3.1 of [1]:

2.13. TheOREM. Under the assumptions of Proposition 2.12 let $S_{1}$ be the set of all points $z_{0} \in E$ for which there is a full bounded solution $z: \mathbb{R} \rightarrow E$ of $f_{K_{1}}$ such that $z(0)=z_{0}$. Then the Conley index $h\left(f_{K_{1}}, S_{1}\right)$ is defined and

$$
h\left(f_{K_{1}}, S_{1}\right)_{l}=\Sigma^{l} \quad \text { for all } l \text { sufficiently large. }
$$

Proof. For $\theta \in[0,1]$ let $S_{\theta}$ be the set of all points $z_{0} \in E$ for which there is a full bounded solution $z: \mathbb{R} \rightarrow E$ of $f_{K_{\theta}}$ such that $z(0)=z_{0}$. By Proposition 2.12 there is a bounded closed set $N \subset E$ such that for every $\theta \in$ $[0,1]$ the set $N$ is an isolating neighborhood of $S_{\theta}$. Since by Proposition 2.3 the union of the sets $K_{\theta}(N)$ for $\theta \in[0,1]$ is relatively compact in $E$ we deduce from the continuation invariance of the Conley index (see Proposition 4.13 of [9]) that

$$
h\left(f_{K_{0}}, N\right)_{l}=h\left(f_{K_{1}}, N\right)_{l} \quad \text { for } l \text { large enough. }
$$

But by Proposition 2.2 and Theorem 2.6 the operator $f_{K_{0}}=L$ is hyperbolic so 0 is the only full bounded solution of $f_{K_{0}}$. Therefore $S_{0}=\{0\}$ and so, by Theorem 2.6 (with $a=b=c=0$ ), we have

$$
h\left(f_{K_{0}}, N\right)_{l}=\Sigma^{l} \quad \text { for all } l \in \mathbb{N} .
$$

Now (2.26) and (2.27) imply the assertion of the corollary.

2.14. Corollary. The set of solutions of system (1.1) is nonempty.

Proof. Since $\left(f_{K_{1}}, E\right)$ is gradient-like, it is enough to prove that $S_{1} \neq \emptyset$. But the index $h\left(f_{K_{1}}, \emptyset\right)$ satisfies, by Proposition 4.12 of [9], the formula $h\left(f_{K_{1}}, \emptyset\right)_{l}=\overline{0}$ for $l$ large enough. Since $\Sigma^{l} \neq \overline{0}$, the corollary follows from Theorem 2.13.

2.15. Corollary. Suppose $z=0$ is an isolated solution of (1.1) such that

$$
h\left(f_{K_{1}},\{0\}\right)_{l} \neq \Sigma^{l} \quad \text { for infinitely many } l \in \mathbb{N} .
$$

Then system (1.1) has a nontrivial solution.

Proof. Again it is sufficient to prove that $S_{1} \neq\{0\}$. But this is clear from (2.28) and Theorem 2.13.

We can now state the following analogue of Angenent and van der Vorst's result on the existence of nontrivial solutions of system (1.1): 
2.16. Theorem (cf. Section 9.2.1 of [1]). Suppose

$$
\partial_{\xi} H(0,0, x)=\partial_{\eta} H(0,0, x) \equiv 0 .
$$

Let $K_{\operatorname{lin}}: E \rightarrow E$ be the map given by

$$
K_{\text {lin }}(u, v)=\left(A^{-2 s}(-a(\cdot) u+c(\cdot) v), A^{-2 t}(c(\cdot) u-b(\cdot) v)\right) .
$$

Here, the continuous functions $a, b, c: \bar{\Omega} \rightarrow \mathbb{R}$ are defined, for $x \in \bar{\Omega}$, by

$$
\begin{gathered}
a(x)=\partial_{\xi \xi} H(0,0, x), \quad b(x)=\partial_{\eta \eta} H(0,0, x), \\
-c(x)=\partial_{\xi \eta} H(0,0, x)=\partial_{\eta \xi} H(0,0, x) .
\end{gathered}
$$

Suppose that the operator $L+K_{\operatorname{lin}}$ is hyperbolic. Assume that the Conley index $h\left(f_{K_{\text {lin }}},\{0\}\right)$ of $\{0\}$ relative to the linear operator $f_{K_{\text {lin }}}$ satisfies

$$
h\left(f_{K_{\text {lin }}},\{0\}\right)_{l} \neq \Sigma^{l} \quad \text { for infinitely many } l \in \mathbb{N} .
$$

Then system (1.1) has a nontrivial solution. Condition (2.29) is, in particular, satisfied if the functions $a, b$ and $c$ are constant, $a b>0$ and there exists $a k \in \mathbb{N}$ such that $\left(\lambda_{k}+c\right)^{2}-a b<0$.

Proof. The theorem is an immediate consequence of Theorem 2.9, Corollary 2.15 and Theorem 2.6.

2.17. Remark. In view of Corollary 2.10, condition (2.29) means that there is an integer $\gamma \neq 0$ such that

$$
h\left(f_{K_{\text {lin }}},\{0\}\right)_{l}=\Sigma^{l+\gamma} \quad \text { for all } l \in \mathbb{N} \text { large enough. }
$$

By Remark 2.11 this means that the renormalized Morse index of $z_{0}=0$ is different from zero. Thus the first part of Theorem 2.16 is exactly the result stated in the first paragraph of Section 9.2.1 of [1].

2.18. Remark. In their paper [1] Angenent and van der Vorst also consider system (1.1) for the so-called "+"-type Hamiltonians. In that case the set of all full bounded orbits of (2.9) is unbounded and so it is not clear whether Conley index theory is applicable as such.

3. Appendix. In this Appendix we prove a few known or essentially known technical results required to establish the results of Section 2.

3.1. Proposition (cf. Proposition 1.2 of [5]). The map

$$
\psi: E \rightarrow \mathbb{R}, \quad(u, v) \mapsto \int_{\Omega} H(u(x), v(x), x) d x,
$$

is well defined, of class $C^{1}$ and it maps bounded sets in $E$ into bounded sets 
in $\mathbb{R}$. Moreover,

$$
\nabla \psi=G
$$

where $G$ is defined in (2.5) and (2.6).

The map $\nabla \psi: E \rightarrow E$ maps bounded sets in $E$ into relatively compact sets in $E$. Moreover, for every $\theta \in[0,1]$ we have $f_{K_{\theta}}=\nabla \Phi_{\theta}$. Furthermore, there is a $C \in] 0, \infty[$ such that for all $z=(u, v) \in E$,

$$
\begin{aligned}
\langle\nabla \psi(z), \bar{z}\rangle & \geq-c_{2} \mu(\Omega)+\delta\left(|u|_{L^{p}}^{p}+|v|_{L^{q}}^{q}\right), \\
|\nabla \psi(z)|_{E} & \leq C\left(g\left(|u|_{L^{p}},|v|_{L^{q}}\right)+1\right),
\end{aligned}
$$

where $\mu(\Omega)$ is the Lebesgue measure of $\Omega$ and $g:[0, \infty[\times[0, \infty[\rightarrow \mathbb{R}$ is defined as $g(x, y)=x^{p-1}+x^{(q-1) p / q}+y^{q-1}+y^{(p-1) q / p}$.

Proof. Hypothesis (1.4) easily implies that there is a constant $\left.c_{3} \in\right] 0, \infty[$ such that

$$
|H(\xi, \eta, x)| \leq c_{3}\left(|\xi|^{p}+|\eta|^{q}+1\right) \quad \text { for all }(\xi, \eta, x) \in \mathbb{R} \times \mathbb{R} \times \bar{\Omega}
$$

(cf. [5], the proof of formula (1.24)).

The estimate (3.4) together with Hypothesis (1.3) implies that the Nemytskiı̌ operator

$$
\widehat{H}: L^{p}(\Omega) \times L^{q}(\Omega) \rightarrow L^{1}(\Omega), \quad(u, v) \mapsto H(u(\cdot), v(\cdot), x),
$$

is well defined and maps bounded sets in $L^{p}(\Omega) \times L^{q}(\Omega)$ into bounded sets in $L^{1}(\Omega)$. A standard application of Hypothesis (1.4), the mean-value theorem and the Lebesgue dominated convergence theorem imply that $\widehat{H}$ is of class $C^{1}$ and for every $(u, v) \in L^{p}(\Omega) \times L^{q}(\Omega)$ the Fréchet derivative $D \widehat{H}(u, v)$ of $\widehat{H}$ at $(u, v)$ is given, for all $\left(u_{1}, v_{1}\right) \in L^{p}(\Omega) \times L^{q}(\Omega)$, by

$$
\begin{aligned}
D \widehat{H}(u, v)\left(u_{1}, v_{1}\right)(x)= & \partial_{\xi} H(u(x), v(x), x) u_{1}(x) \\
& +\partial_{\eta} H(u(x), v(x), x) v_{1}(x), \quad x \in \Omega .
\end{aligned}
$$

This together with (2.2) implies that the map $\psi$ is well defined, of class $C^{1}$ and it maps bounded sets in $E$ into bounded sets in $\mathbb{R}$. A simple calculation shows formula (3.1).

The compactness of the imbeddings in (2.2) shows that $\nabla \psi=G$ maps bounded sets in $E$ into relatively compact sets in $E$.

Now Hypothesis (1.5) immediately implies the estimate (3.2) while Hypothesis (1.4) implies the existence of a constant $C \in] 0, \infty[$ such that estimate (3.3) holds. The proof is complete.

Proof of Proposition 2.3. This is an immediate consequence of Proposition 3.1 .

Proof of Proposition 2.4. The proposition is essentially contained in the results stated in Section 3.4 of [1]. However, not many details are given there and some of the hints appear to be erroneous. Therefore we give a 
more detailed proof, based on discrete dynamical systems. The method of the proof is of independent interest and it appears to be applicable to a variety of problems.

Set

$$
x^{*}=1 / 2-s / N \text { and } y^{*}=1 / 2-t / N .
$$

We first claim that

(3.7) there are numbers $p^{\prime}$ and $q^{\prime}$ with $1 / p^{\prime}>x^{*},\left(1 / q^{\prime}\right)>y^{*}$ such that

$$
\begin{array}{rlrl}
p^{\prime}-1 & >\max (p-1,1), & \left(p^{\prime}-1\right) q^{\prime} / p^{\prime}>\max ((p-1) q / p, 1), \\
q^{\prime}-1>\max (q-1,1), & \left(q^{\prime}-1\right) p^{\prime} / q^{\prime}>\max ((q-1) p / q, 1) .
\end{array}
$$

In fact, if $x^{*}>0$ and $y^{*}>0$ then $p^{*}:=1 / x^{*}$ and $q^{*}:=1 / y^{*}$ satisfy (3.8) (with $p^{\prime}$ and $q^{\prime}$ replaced by $p^{*}$ and $q^{*}$ respectively), so choosing $p^{\prime}<p^{*}$ close to $p^{*}$ and $q^{\prime}<q^{*}$ close to $q^{*}$ we see that (3.7) is satisfied. If $x^{*} \leq 0$ and $y^{*}>0$ (resp. if $x^{*}>0$ and $y^{*} \leq 0$ ) then by taking $p^{\prime}$ large enough and $q^{\prime}<q^{*}$ close to $q^{*}$ (resp. $q^{\prime}$ large enough and $p^{\prime}<p^{*}$ close to $p^{*}$ ) we see that (3.7) is satisfied. Finally, if $x^{*} \leq 0$ and $y^{*} \leq 0$ then all $p^{\prime}$ and $q^{\prime}$ large enough satisfy (3.7).

Now using (3.7), replacing $p$ by $p^{\prime}$ and $q$ by $q^{\prime}$, and changing the constant $c$ in (2.8) if necessary, we can assume that

$$
\min (p-1,(p-1) q / p, q-1,(q-1) p / q) \geq 1
$$

and for all $i \in I$ and all $(\xi, \eta, x) \in \mathbb{R} \times \mathbb{R} \times \bar{\Omega}$,

$$
\begin{aligned}
& \left|\pi_{i}(\xi, \eta, x)\right| \leq c\left(|\xi|^{p-1}+|\eta|^{(p-1) q / p}+1\right), \\
& \left|\varrho_{i}(\xi, \eta, x)\right| \leq c\left(|\eta|^{q-1}+|\xi|^{(q-1) p / q}+1\right) .
\end{aligned}
$$

It follows from (3.10) that

(3.11) whenever $i \in I, p_{1}, q_{1} \in\left[1, \infty\left[\right.\right.$ and $(u, v) \in L^{p_{1}}(\Omega) \times L^{q_{1}}(\Omega)$ then $\pi_{i}(u(\cdot), v(\cdot), \cdot) \in L^{q_{2}}(\Omega)$ and $\varrho_{i}(u(\cdot), v(\cdot), \cdot) \in L^{p_{2}}(\Omega)$, where the numbers $p_{2}$ and $q_{2}$ are defined by

$$
\begin{aligned}
& 1 / p_{2}=\max \left((q-1) / q_{1},(q-1) p /\left(q p_{1}\right)\right), \\
& 1 / q_{2}=\max \left((p-1) / p_{1},(p-1) q /\left(p q_{1}\right)\right) .
\end{aligned}
$$

In particular, for every $(u, v) \in E \subset L^{p}(\Omega) \times L^{q}(\Omega)$ and $i \in I$ we find that $\pi_{i}(u(\cdot), v(\cdot), \cdot) \in L^{p /(p-1)}$ and $\varrho_{i}(u(\cdot), v(\cdot), \cdot) \in L^{q /(q-1)}$. Thus, proceeding as in the proof of Theorem 1.2 of [5] we see that whenever $i \in I$ and $z=(u, v) \in E$ is such that

$$
L z+\left(A^{-2 s} \pi_{i}(u(\cdot), v(\cdot), \cdot), A^{-2 t} \varrho_{i}(u(\cdot), v(\cdot), \cdot)\right)=0,
$$

then

$$
u=-B_{q /(q-1)}^{-1} \varrho_{i}(u(\cdot), v(\cdot), \cdot), \quad v=-B_{p /(p-1)}^{-1} \pi_{i}(u(\cdot), v(\cdot), \cdot) .
$$


Here, for $r \in\left[0, \infty\left[\right.\right.$ the map $B_{r}$ is defined by

$$
B_{r}: W_{0}^{1, r}(\Omega) \cap W^{2, r}(\Omega) \rightarrow L^{r}(\Omega), \quad u \mapsto-\Delta u .
$$

Notice that $B=B_{2}$, where $B$ was defined at the beginning of Section 2 . Also note that if $r<r^{\prime}$ and $w \in L^{r^{\prime}}(\Omega) \subset L^{r}(\Omega)$ then

$$
B_{r^{\prime}}^{-1} w=B_{r}^{-1} w
$$

and so we will drop the subscript altogether, writing $B^{-1}$ instead of $B_{r}^{-1}$, for every $r \in[1, \infty[$. Thus, by the usual Sobolev inequalities and Schauder estimates (cf. [7]),

(3.13) for every $r \in\left[1, \infty\left[\right.\right.$ with $1 / r-2 / N>0$ there is a constant $C_{r} \in$ $\left[0, \infty\left[\right.\right.$ such that whenever $w \in L^{r}(\Omega)$ then $B^{-1} w \in L^{r^{\prime}}(\Omega)$ and $\left|B^{-1} w\right|_{L^{r^{\prime}}} \leq C_{r}|w|_{L^{r}}$ where $1 / r^{\prime}=1 / r-2 / N$,

(3.14) for every $r \in\left[1, \infty\left[\right.\right.$ with $1 / r-2 / N<0$ there is a constant $C_{r} \in$ $\left[0, \infty\left[\right.\right.$ such that whenever $w \in L^{r}(\Omega)$ then $B^{-1} w \in C^{\nu}(\bar{\Omega})$ and $\left|B^{-1} w\right|_{C^{\nu}} \leq C_{r}|w|_{L^{r}}$ where $\nu=2-N / r$.

In particular,

$$
\begin{aligned}
& \text { for all } p_{0}, q_{0} \in[1, \infty[\text { with } \\
& \qquad(q-1) / q-2 / N \leq 1 / p_{0}, \quad(p-1) / p-2 / N \leq 1 / q_{0},
\end{aligned}
$$

there is a constant $C_{p_{0}, q_{0}} \in[0, \infty[$ such that whenever $z \in Z$ then

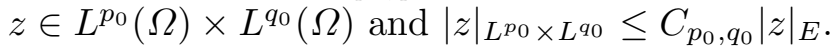

By perturbing $s$ a little, we may suppose that $x^{*} \neq 0$ and $y^{*} \neq 0$.

Assume first that

$$
x^{*}>0 \text { and } y^{*}>0 .
$$

Since $1 / p>x^{*}$ and $1 / q>y^{*}$, we obtain

$$
\begin{array}{ll}
p-1<(N+2 s) /(N-2 s), & q-1<(N+2 t) /(N-2 t), \\
(p-1) q / p<(N+2 s) /(N-2 t), & (q-1) p / q<(N+2 t) /(N-2 s) .
\end{array}
$$

We will need the following lemma.

3.2. Lemma. Define the map $\Gamma: \mathbb{R}^{2} \rightarrow \mathbb{R}^{2}$ by

$$
\Gamma(x, y)=\left(\Gamma_{1}(x, y), \Gamma_{2}(x, y)\right), \quad(x, y) \in \mathbb{R}^{2},
$$

where

$$
\begin{aligned}
& \Gamma_{1}(x, y)=\max ((q-1) y,(q-1) p x / q)-2 / N, \\
& \Gamma_{2}(x, y)=\max ((p-1) x,(p-1) q y / p)-2 / N .
\end{aligned}
$$

Let $U$ be the set of all $(x, y) \in \mathbb{R}^{2}$ with $x \leq x^{*}$ and $y \leq y^{*}$. Then:

$$
\text { Whenever }(x, y) \in U \text {, then } \Gamma_{1}(x, y)<x^{*} \text { and } \Gamma_{2}(x, y)<y^{*} \text {. }
$$


(3.20) For every $(\bar{x}, \bar{y}) \in U$ there are $n=n(\bar{x}, \bar{y}) \in \mathbb{N}$ and $k \in\{1,2\}$ such that the $k$ th component $\left(\Gamma^{n}(\bar{x}, \bar{y})\right)_{k}$ of $\Gamma^{n}(\bar{x}, \bar{y})$ satisfies

$$
\left(\Gamma^{n}(\bar{x}, \bar{y})\right)_{k}<0
$$

where, as usual, $\Gamma^{n}$ denotes the $n$th iterate of the function $\Gamma$.

Moreover, there is a $\delta=\delta(\bar{x}, \bar{y}) \in] 0, \infty[$ such that whenever $|(x, y)-(\bar{x}, \bar{y})|$ $\leq \delta, x<\bar{x}$ and $y<\bar{y}$, then $\left(\Gamma^{m}(x, y)\right)_{j} \neq 0$ for all $m \in \mathbb{N}$ with $m \leq n$ and $j=1,2$.

Assume the lemma for a moment. Note that $(q-1) / q-2 / N<x^{*}$ and $(p-1) / p-2 / N<y^{*}$. Let $x_{0}>0, y_{0}>0,(q-1) / q-2 / N<x_{0}<x^{*}$ and $(p-1) / p-2 / N<y_{0}<y^{*}$. Set $\left(x_{n}, y_{n}\right)=\Gamma^{n}\left(x_{0}, y_{0}\right), n \in \mathbb{N}$. Perturbing $\left(x_{0}, y_{0}\right)$ a little and using Lemma 3.2 we may assume that there are $n \in \mathbb{N}$ and $j \in\{1,2\}$ such that

$$
x_{m}>0 \text { and } y_{m}>0 \text { for } m \in \mathbb{N}_{0} \text { with } m \leq n-1, x_{n} \neq 0, y_{n} \neq 0
$$
and, finally, $x_{n}<0$ or $y_{n}<0$ (or both).

Set $p_{m}=1 / x_{m}$ and $q_{m}=1 / y_{m}$ for all $m \in \mathbb{N}_{0}$ with $m \leq n$.

Suppose, e.g., that $x_{n}<0$ and $y_{n}>0$. Note that $2-x_{n-1} N>0$, since otherwise $x_{n} \geq 0$ by the definition of $\Gamma$, and choose $\left.\beta \in\right] 0,2-x_{n-1} N$, $\beta<1$, arbitrarily.

Using (3.15) we see that whenever $z \in Z$ with $|z|_{E} \leq C^{\prime}$ then $z \in$ $L^{p_{0}}(\Omega) \times L^{q_{0}}(\Omega)$ with $|z|_{L^{p_{0}} \times L^{q_{0}}} \leq C^{\prime \prime}\left(C^{\prime}\right)$.

Using (3.13) and (3.11) we see that whenever $m \in \mathbb{N}_{0}$ with $m \leq n-2$ and $z \in Z \cap\left(L^{p_{m}}(\Omega) \times L^{q_{m}}(\Omega)\right)$ with $|z|_{L^{p_{m}} \times L^{q_{m}}} \leq C^{\prime}$ then $z \in L^{p_{m+1}}(\Omega) \times$ $L^{q_{m+1}}(\Omega)$ with $|z|_{L^{p_{m+1}} \times L^{q_{m+1}}} \leq C^{\prime \prime}\left(C^{\prime}\right)$.

Using (3.14) and (3.11) we see that whenever $z \in Z \cap\left(L^{p_{n-1}}(\Omega) \times\right.$ $\left.L^{q_{n-1}}(\Omega)\right)$ with $|z|_{L^{p_{n-1}} \times L^{q_{n-1}}} \leq C^{\prime}$ then $z \in C^{\beta}(\bar{\Omega}) \times L^{q_{n}}(\Omega)$ with $|z|_{C^{\beta} \times L^{q_{n}}}$ $\leq C^{\prime \prime}\left(C^{\prime}\right)$.

Let $c^{\prime} \in\left[0, \infty\left[\right.\right.$. It follows that there is a constant $\left.c_{1}^{\prime}=c_{1}^{\prime}\left(c^{\prime}\right) \in\right] 0, \infty[$ such that whenever $z=(u, v) \in Z$ is such that $|z|_{E} \leq c^{\prime}$ then $u \in C^{\beta}(\bar{\Omega})$, $|u|_{C^{\beta}} \leq c_{1}^{\prime}, v \in L^{q_{n}}(\Omega)$ and $|v|_{L^{q_{n}}} \leq c_{1}^{\prime}$. Consequently,

$$
\left|\pi_{i}(u(x), v(x), x)\right| \leq c_{2}^{\prime}\left(|v(x)|^{(p-1) q / p}+1\right)
$$

for some constant $c_{2}^{\prime}=c_{2}^{\prime}\left(c, c_{1}^{\prime}\right) \geq c_{1}^{\prime}$, independent of $i$ and $z \in Z$. Using (3.22), the arguments leading to the proof of estimate (3.24) below and the above arguments concerning the function $u$, we see that $v \in C^{\beta}(\bar{\Omega})$ and we may assume that the constants $c_{2}^{\prime}$ and $\beta$ are such that $|v|_{C^{\beta}} \leq c_{2}^{\prime}$.

Since the functions $\pi_{i}$ and $\varrho_{i}$ are of class $C^{1}$, it follows that the functions $\pi_{i}(u(\cdot), v(\cdot), \cdot)$ and $\varrho_{i}(u(\cdot), v(\cdot), \cdot)$ are $\beta$-Hölder continuous. Using Schauder estimates once again we find that $u, v \in C_{0}^{2, \beta}(\bar{\Omega})$ and we may assume that the constant $c_{2}^{\prime}$ is such that $|z|_{C^{2, \beta} \times C^{2, \beta}} \leq c_{2}^{\prime}$. Setting $c^{\prime \prime}=c_{2}^{\prime}$ we complete the proof of the proposition for $x_{n+1}<0, y_{n+1}>0$. 
The proof in case $y_{n+1}<0$ is analogous. Therefore the proposition is proved for $x^{*}>0, y^{*}>0$. The remaining case, $x^{*}<0$ or $y^{*}<0$, is easier since we then immediately have uniform estimates for one of the component functions $u$ or $v$ of $z$, and so we only have to bootstrap with respect to either $u$ or $v$. Thus we only need to use arguments leading to the proof of estimates (3.23) and (3.24) below. Simple details are left to the reader.

Proof of Lemma 3.2. The proof of (3.19) is a simple computation using (3.6) and (3.17).

Let us prove (3.20). To this end let $(\bar{x}, \bar{y}) \in U$ and define $\left(x_{n}, y_{n}\right)=$ $\Gamma^{n}(\bar{x}, \bar{y}), n \in \mathbb{N}$.

Define the maps $\Gamma^{\prime}: \mathbb{R}^{2} \rightarrow \mathbb{R}^{2}$ and $\Gamma^{\prime \prime}: \mathbb{R}^{2} \rightarrow \mathbb{R}^{2}$ by

$$
\begin{aligned}
\Gamma^{\prime}(x, y) & =((q-1) p x / q-2 / N,(p-1) x-2 / N), & (x, y) \in \mathbb{R}^{2}, \\
\Gamma^{\prime \prime}(x, y) & =((q-1) y-2 / N,(p-1) q y / p-2 / N), & (x, y) \in \mathbb{R}^{2} .
\end{aligned}
$$

It follows that

$$
\Gamma(x, y)= \begin{cases}\Gamma^{\prime}(x, y) & \text { for } p x \geq q y \\ \Gamma^{\prime \prime}(x, y) & \text { for } p x<q y\end{cases}
$$

Let a $\Gamma^{\prime}$-segment of length $k$ be any finite sequence $\left(x_{m}, y_{m}\right)_{m=j}^{j+k}$ such that $\left(x_{m+1}, y_{m+1}\right)=\Gamma^{\prime}\left(x_{m}, y_{m}\right)$ for all $m=j, \ldots, j+k-1$. Define $\Gamma^{\prime \prime}{ }_{-}$ segments of length $k$ analogously.

Using (3.9) we see that whenever $(x, y) \in U$ then

$$
\begin{aligned}
\Gamma_{1}^{\prime}(x, y)-x & =((q-1) p / q-1) x-2 / N \\
& \leq((q-1) p / q-1) x^{*}-2 / N=:-\alpha
\end{aligned}
$$

and

$$
\begin{aligned}
\Gamma_{2}^{\prime \prime}(x, y)-y & =((p-1) q / p-1) y-2 / N \\
\leq & ((p-1) q / p-1) y^{*}-2 / N=:-\beta .
\end{aligned}
$$

Using (3.17) we immediately see that $\alpha>0$ and $\beta>0$. Thus

$$
\begin{gathered}
\Gamma_{1}^{\prime}(x, y)-x \leq-\alpha<0 \\
\Gamma_{2}^{\prime \prime}(x, y)-y \leq-\beta<0
\end{gathered}
$$

Now suppose that $j, k, l \in \mathbb{N}$ are such that $\left(x_{m}, y_{m}\right)_{m=j}^{j+k}$ is a $\Gamma^{\prime}$-segment and $\left(x_{m}, y_{m}\right)_{m=j+k+1}^{j+k+l}$ is a $\Gamma^{\prime \prime}$-segment. Then, by (3.23) and (3.24),

$$
\begin{aligned}
x_{j+k+l} & =(q-1) y_{j+k+l-1}-2 / N \leq(q-1) y_{j+k}-2 / N \\
& =(q-1)\left((p-1) x_{j+k-1}-2 / N\right)-2 / N \\
& \leq(q-1)\left((p-1) x_{j}-2 / N\right)-2 / N .
\end{aligned}
$$


Hence

$$
\begin{aligned}
x_{j+k+l}-x_{j} & \leq(q-1)\left((p-1) x_{j}-2 / N\right)-2 / N-x_{j} \\
& =((q-1)(p-1)-1) x_{j}-(q-1)(2 / N)-2 / N \\
& \leq((q-1)(p-1)-1) x^{*}-(q-1)(2 / N)-2 / N=:-\gamma .
\end{aligned}
$$

Using (3.17) we immediately see that $\gamma>0$. Thus

$$
x_{j+k+l}-x_{j} \leq-\gamma .
$$

Let $\bar{k} \in \mathbb{N}$ be such that $\bar{k} \alpha>x^{*}$ and $\bar{k} \beta>y^{*}$.

If the sequence $\left(x_{n}, y_{n}\right)_{n \in \mathbb{N}}$ contains a $\Gamma^{\prime}$-segment $\left(x_{m}, y_{m}\right)_{m=j}^{j+\bar{k}}$ of length $\bar{k}$ it follows from (3.23) that $x_{j+\bar{k}} \leq x_{j}-\bar{k} \alpha \leq x^{*}-\bar{k} \alpha<0$ and the first part of (3.20) is proved.

Similarly, if $\left(x_{n}, y_{n}\right)_{n \in \mathbb{N}}$ contains a $\Gamma^{\prime \prime}$-segment $\left(x_{m}, y_{m}\right)_{m=j}^{j+\bar{k}}$ of length $\bar{k}$ then, by (3.24), $y_{j+\bar{k}}<0$ and the first part of (3.20) is proved again.

If $\left(x_{n}, y_{n}\right)_{n \in \mathbb{N}}$ does not contain any $\Gamma^{\prime}$ - or $\Gamma^{\prime \prime}$-segment of length $\bar{k}$ then there are sequences $\left(k_{r}\right)_{r \in \mathbb{N}}$ and $\left(l_{r}\right)_{r \in \mathbb{N}}$ in $\mathbb{N}$ with $k_{r}<\bar{k}$ and $l_{r}<\bar{k}$ for all $r \in \mathbb{N}$ and there is an $\bar{n} \in \mathbb{N}$ such that the sequence $\left(x_{n}, y_{n}\right)_{n \geq \bar{n}}$ is made up of a $\Gamma^{\prime}$-segment of length $k_{1}$ followed by a $\Gamma^{\prime \prime}$-segment of length $l_{1}$ followed by a $\Gamma^{\prime}$-segment of length $k_{2}$ and so on.

Let $\bar{r} \in \mathbb{N}$ be such that $\bar{r} \gamma>x^{*}$. Let $n=\bar{n}+\sum_{r=1}^{\bar{r}}\left(k_{r}+l_{r}\right)$. It follows from (3.25) that $x_{n} \leq x_{\bar{n}}-\bar{r} \gamma \leq x^{*}-\bar{r} \gamma<0$. This again proves the first part of (3.20).

The last statement in (3.20) follows immediately from the continuity of $\Gamma$ and the fact that $\Gamma$ is strictly increasing, i.e. whenever $x<x^{\prime}$ and $y<y^{\prime}$ then $\Gamma_{j}(x, y)<\Gamma_{j}\left(x^{\prime}, y^{\prime}\right)$ for $j=1,2$.

For the rest of this section we write $f_{\theta}:=f_{K_{\theta}}, \theta \in[0,1]$.

3.3. Proposition. Let $C$ be as in Proposition 3.1. For every $\alpha \in[0, \delta / C[$ there is a $\left.d_{\alpha} \in\right] 0, \infty\left[\right.$ such that whenever $z \in E, \theta \in[0,1]$ and $\left|\nabla \Phi_{\theta}(z)\right|_{E} \leq$ $\alpha$, then $|z|_{E}<d_{\alpha}$.

Proof. In this proof we write $|\cdot|$ for $|\cdot|_{E}$. The definition of the function $g$ implies that there is a $w \in] 0, \infty[$ such that

$$
\text { whenever } x, y \in] 0, \infty\left[\text { and } g(x, y) \geq w \text { then } g(x, y) \leq x^{p}+y^{q} .\right.
$$

Let $\alpha \in[0, \delta / C[$. Set

$$
d^{\prime}:=\alpha+C(w+1), \quad d^{\prime \prime}=(\delta / C-\alpha)^{-1}\left(\alpha \delta / C+\delta+c_{2} \mu(\Omega)\right) .
$$

Then $\left.d=d_{\alpha}:=\max \left(d^{\prime}, d^{\prime \prime}\right) \in\right] 0, \infty[$. Let $z=(u, v) \in E$ and $\theta \in[0,1]$ with $\left|\nabla \Phi_{\theta}(z)\right| \leq \alpha$. We need to show that $|z|<d_{\alpha}$. If $|z|<d^{\prime}$ then we are done, so suppose that $|z| \geq d^{\prime}$. Then 


$$
\begin{aligned}
d^{\prime} & \leq|z|=|L z| \leq|L z-\theta \nabla \psi(z)|+|\theta \nabla \psi(z)|=\left|\nabla \Phi_{\theta}(z)\right|+\theta|\nabla \psi(z)| \\
& \leq \alpha+\theta C\left(g\left(|u|_{L^{p}},|v|_{L^{q}}\right)+1\right) \leq \alpha+C\left(g\left(|u|_{L^{p}},|v|_{L^{q}}\right)+1\right) .
\end{aligned}
$$

The definition of $d^{\prime}$ implies that $g\left(|u|_{L^{p}},|v|_{L^{q}}\right) \geq w$, so by (3.26) we obtain $g\left(|u|_{L^{p}},|v|_{L^{q}}\right) \leq|u|_{L^{p}}^{p}+|v|_{L^{q}}^{q}$. Hence

$$
|z| \leq \alpha+\theta C\left(|u|_{L^{p}}^{p}+|v|_{L^{q}}^{q}\right)+\theta C .
$$

Now

$$
\begin{aligned}
\alpha|z| & =\alpha|\bar{z}| \geq\left\langle-\nabla \Phi_{\theta}(z), \bar{z}\right\rangle=-\langle L z, \bar{z}\rangle+\theta\langle\nabla \psi(z), \bar{z}\rangle=\theta\langle\nabla \psi(z), \bar{z}\rangle \\
& \geq-c_{2} \theta \mu(\Omega)+\delta \theta\left(|u|_{L^{p}}^{p}+|v|_{L^{q}}^{q}\right)
\end{aligned}
$$

SO

$$
\alpha|z| \geq-c_{2} \theta \mu(\Omega)+\delta \theta\left(|u|_{L^{p}}^{p}+|v|_{L^{q}}^{q}\right) .
$$

Using (3.27) and (3.28) we obtain

$$
|z| \leq \alpha+(C / \delta) \delta \theta\left(|u|_{L^{p}}^{p}+|v|_{L^{q}}^{q}\right)+\theta C \leq \alpha+(C / \delta)\left(\alpha|z|+c_{2} \theta \mu(\Omega)\right)+\theta C
$$

so, by the definition of $d^{\prime \prime},|z| \leq d^{\prime \prime}$ and hence $|z| \leq d_{\alpha}$, as claimed.

Proof of Proposition 2.12. We again write $|\cdot|$ instead of $|\cdot|_{E}$. Let $\alpha \in\left[0, \delta / C\left[\right.\right.$, and let $\left.d:=d_{\alpha} \in\right] 0, \infty[$ be as in Proposition 3.3. Let $C^{\prime}:=\sup \left\{\Phi_{\theta}(z) \mid \theta \in[0,1]\right.$ and $\left.|z|<d\right\}$. It follows from Proposition 3.1 that $\left.C^{\prime} \in\right] 0, \infty[$.

Suppose Proposition 2.12 is false. Then there are sequences $\left(\theta_{n}\right)$ in $[0,1]$ and $\left(z_{n}\right)$ such that

(3.29) for every $n \in \mathbb{N}, z_{n}$ is a full bounded solution of $f_{\theta_{n}}$ satisfying $z_{n}(0) \geq n+d$.

Proposition 4.3 of $[9]$ implies that for every $n \in \mathbb{N}$ the set $z_{n}(\mathbb{R})$ is relatively compact in $E$. Since $\left(f_{\theta_{n}}, E\right)$ is gradient-like, it follows that both $\alpha\left(z_{n}\right)$ and $\omega\left(z_{n}\right)$ contain only equilibria of $f_{\theta_{n}}$. Thus $y \in \alpha\left(z_{n}\right) \cup \omega\left(z_{n}\right)$ implies that $\nabla \Phi_{\theta_{n}}(y)=0$, in particular $\left|\nabla \Phi_{\theta_{n}}(y)\right| \leq \alpha$ so $|y|<d$ and so $\left|\Phi_{\theta_{n}}(y)\right| \leq C^{\prime}$. Since $\Phi_{\theta_{n}}$ is nondecreasing along the solution $z_{n}$ we obtain

$$
-C^{\prime} \leq \Phi_{\theta_{n}}\left(z_{n}(\tau)\right) \leq C^{\prime}, \quad \tau \in \mathbb{R} .
$$

Now (3.29) and (3.30) imply that for all $n \in \mathbb{N}$ large enough there are $\left.\left.a_{n} \in\right]-\infty, 0\right]$ and $b_{n} \in[0, \infty[$ such that

$$
\begin{aligned}
& \left.\left.a_{n}=\max \{\tau \in]-\infty, 0\right] \mid z_{n}(\tau)=d\right\}, \\
& b_{n}=\min \left\{\tau \in \left[0, \infty\left[\mid z_{n}(\tau)=d\right\} .\right.\right.
\end{aligned}
$$

We thus obtain 


$$
\begin{aligned}
& n \leq\left|z_{n}(0)\right|-\left|z_{n}\left(b_{n}\right)\right| \leq\left|z_{n}\left(b_{n}\right)-z_{n}(0)\right| \leq \int_{0}^{b_{n}}\left|f_{\theta_{n}}\left(z_{n}(\tau)\right)\right| d \tau \\
& n \leq\left|z_{n}(0)\right|-\left|z_{n}\left(a_{n}\right)\right| \leq\left|z_{n}(0)-z_{n}\left(a_{n}\right)\right| \leq \int_{a_{n}}^{0}\left|f_{\theta_{n}}\left(z_{n}(\tau)\right)\right| d \tau .
\end{aligned}
$$

Setting $I_{n}:=\left[a_{n}, b_{n}\right]$ we see that

$$
\int_{I_{n}}\left|f_{\theta_{n}}\left(z_{n}(\tau)\right)\right| d \tau \rightarrow \infty \quad \text { as } n \rightarrow \infty .
$$

Now, for every $n \in \mathbb{N}$ and $\tau \in \mathbb{R}$ we have

$$
\left|f_{\theta_{n}}\left(z_{n}(\tau)\right)\right|^{2}=\left\langle f_{\theta_{n}}\left(z_{n}(\tau)\right), \dot{z}_{n}(\tau)\right\rangle=\left(\Phi_{\theta_{n}} \circ z_{n}\right)^{\prime}(\tau)
$$

and so

$$
\int_{I_{n}}\left|f_{\theta_{n}}\left(z_{n}(\tau)\right)\right|^{2} d \tau=\Phi_{\theta_{n}}\left(z_{n}\left(b_{n}\right)\right)-\Phi_{\theta_{n}}\left(z_{n}\left(a_{n}\right)\right) \leq 2 C^{\prime} .
$$

If $\tau \in I_{n}$, then by (3.31) we have $\left|z_{n}(\tau)\right| \geq d$, which implies $\left|f_{\theta_{n}}\left(z_{n}(\tau)\right)\right| \geq \alpha$, so

$$
\int_{I_{n}}\left|f_{\theta_{n}}\left(z_{n}(\tau)\right)\right|^{2} d \tau \geq \alpha^{2}\left(b_{n}-a_{n}\right)
$$

and thus, by (3.33),

$$
\alpha^{2}\left(b_{n}-a_{n}\right) \leq 2 C^{\prime}
$$

Setting $J_{n}:=\left\{\tau \in I_{n}|| f_{\theta_{n}}\left(z_{n}(\tau)\right) \mid \geq 1\right\}$ we thus have, for every $n \in \mathbb{N}$,

$$
\begin{aligned}
\int_{I_{n}}\left|f_{\theta_{n}}\left(z_{n}(\tau)\right)\right| d \tau & =\int_{J_{n}}\left|f_{\theta_{n}}\left(z_{n}(\tau)\right)\right| d \tau+\int_{I_{n} \backslash J_{n}}\left|f_{\theta_{n}}\left(z_{n}(\tau)\right)\right| d \tau \\
& \leq \int_{J_{n}}\left|f_{\theta_{n}}\left(z_{n}(\tau)\right)\right|^{2} d \tau+\left(b_{n}-a_{n}\right) \leq 2 C^{\prime}+2 C^{\prime} \alpha^{-2}<\infty .
\end{aligned}
$$

However, this contradicts (3.32) and proves the proposition.

3.4. RemarK. Notice that Hypothesis (1.5) is required only in Proposition 2.12, Theorem 2.13, Corollaries 2.14 and 2.15, Theorem 2.16 and Proposition 3.3 .

\section{References}

[1] S. Angenent and R. van der Vorst, A superquadratic indefinite elliptic system and its Morse-Conley-Floer homology, Math. Z. 231 (1999), 203-248.

[2] -, - A priori bounds and generalized Morse indices of solutions of an elliptic system, Ann. Inst. H. Poincaré, to appear.

[3] M. C. Carbinatto and K. P. Rybakowski, Morse decompositions in the absence of uniqueness, Topol. Methods Nonlinear Anal., in press. 
[4] C. C. Conley, Isolated Invariant Sets and the Morse Index, CBMS Regional Conf. Ser. in Math. 38, Amer. Math. Soc., Providence, 1978.

[5] D. G. de Figueiredo and P. L. Felmer, On superquadratic elliptic systems, Trans. Amer. Math. Soc. 343 (1994), 99-116.

[6] K. Gęba, M. Izydorek and A. Pruszko, The Conley index in Hilbert spaces, Studia Math. 134 (1999), 217-233.

[7] D. Gilbarg and N. S. Trudinger, Elliptic Partial Differential Equations of Second Order, Springer, Berlin, 1983.

[8] D. Henry, Geometric Theory of Semilinear Parabolic Equations, Lecture Notes in Math. 840, Springer, New York, 1981.

[9] M. Izydorek and K. P. Rybakowski, On the Conley index in Hilbert spaces in the absence of uniqueness, Fund. Math. 171 (2002), 31-52.

[10] - - -, Multiple solutions of indefinite elliptic systems via a Galerkin-type Conley index theory, in preparation.

[11] K. P. Rybakowski, The Homotopy Index and Partial Differential Equations, Springer, Berlin, 1987.

Technical University Gdańsk

Faculty of Technical Physics

and Applied Mathematics

Narutowicza 11/12

80-952 Gdańsk, Poland

E-mail: izydorek@mifgate.pg.gda.pl

Fachbereich Mathematik

Universität Rostock

Universitätsplatz 1

18055 Rostock, Germany

E-mail: krzysztof.rybakowski@mathematik.uni-rostock.de

Received 31 July 2001;

in revised form 5 November 2001 